\title{
Autonomía económica de las mujeres: un reto para el Estado (Estudio de caso, el departamento de Nariño)
}

"Economic autonomy of women: a challenge for
the State (Case study, the department of Nariño)

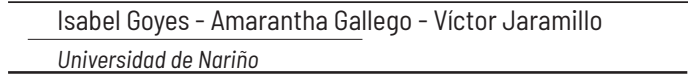

\section{Resumen}

Este artículo somete a discusión los resultados derivados de la revisión normativa colombiana que refuerza el derecho de las mujeres a la autonomía económica, así como la necesidad inaplazable de garantizar la igualdad de género, como una premisa fundamental para avanzar hacia el desarrollo y la justicia social. De igual manera se presentan los resultados de los análisis de la GEIH, mediante los cuales se identificaron las principales barreras y restricciones que enfrentan las mujeres para acceder al mercado laboral, relacionadas con la vulnerabilidad a las cuales están expuestas por el hecho de ser mujeres.

Palabras clave: autonomía económica, igualdad de género, cultura patriarcal, mercado laboral.

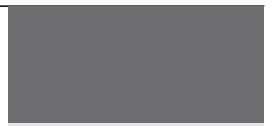

\section{Abstract}

This article aims to discuss the results derived from the Colombian regulatory review that reinforces the right of women to economic autonomy, as well as the urgent need to guarantee gender equality, as a fundamental premise to advance towards development and justice social. Likewise, the results of the GEIH analyzes are presented, through which the main barriers and restrictions faced by women to access the labor market, related to the vulnerability to which they are exposed due to being women, were identified.

Keywords: economic autonomy, gender equality, patriarchal culture, labor market.

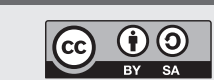

() 2019

\section{Correspondencia de}

autor:

autorlarticulo@correo.com autor2articulo@correo. com autor3articulo@correo. com

\section{Recibido:}

19/Septiembre/2018

\section{Aceptado:}

$11 /$ Novimbre/2018

Publicado:

3/Enero/2019 


\section{Introducción}

Una revisión documental permite afirmar que, durante los últimos 30 años, en Colombia ha existido un gran avance en materia normativa frente a la equidad de género. Gran parte de esta legislación responde tanto a compromisos internacionales, como a procesos sociales de presión protagonizados por colectivos de mujeres frente al Estado, unos y otros han logrado la expedición de leyes tendientes a la reivindicación de los derechos de las mujeres y superar las problemáticas específicas que enfrentan en diversos aspectos, políticos, sociales, familiares, labores y especialmente económicos (Mora \& Caicedo, 2013). No obstante, a pesar de este avance en materia legislativa, aún se mantiene la posición desigual de la mujer frente al hombre, especialmente en materia de trabajo, donde la discriminación laboral y la segregación ocupacional, sumadas a los problemas de precariedad laboral, han afectado la autonomía económica de la mujer y su empoderamiento.

Bajo el escenario anterior, intentar una respuesta frente a interrogantes como: ¿Cuáles es la situación actual de las brechas de género en el mercado de trabajo? ¿Cómo afecta el mercado laboral a la autonomía económica de la mujer? y ¿Qué retos tiene el Estado frente a la autonomía económica?, posibilitará develar un contexto definido por estructuras de restricción que limitan el acceso de la mujer a los recursos productivos y financieros en igualdad de condiciones que los hombres (Kabeer, 2012), escenario que confirma la existencia de una fisura histórica entre la evidencia empírica y la legislación.

En este artículo se analiza la autonomía económica de la mujer desde el mercado de trabajo, profundizando en el caso del departamento de Nariño, región ubicada al suroccidente de Colombia, con un 49,9\% de población femenina (DANE, 2017). La hipótesis que ordena este documento asegura que la existencia de brechas por sexo dentro de los principales indicadores de mercado laboral en el departamento, demuestra la existencia de restricciones, extrínsecas e intrínsecas impuestas (Kabeer, 2012), que impiden a la mujer lograr su autonomía económica, situación que se encuentra ligada a la imposibilidad del Estado para fomentar mecanismos efectivos que permitan superar los estereotipos de género, causantes de los procesos de discriminación laboral, segregación ocupacional y precariedad laboral. 
Frente a la metodología utilizada, se priorizó el análisis documental y cuantitativo. Frente al primero, bajo los lineamientos de López (2005), se realizó una revisión normativa, bajo la técnica de la ubicación de las leyes en un contexto social y político, extrayendo su finalidad y su contenido. Mientras que para el estudio cuantitativo se utilizó un análisis descriptivo y econométrico con el fin de determinar las principales brechas de género y los determinantes de estas brechas. Los datos utilizados en este trabajo fueron tomados de la Gran Encuesta Integrada de Hogares (GEIH), los cuales, siguiendo las recomendaciones de Red Ormet (2015), DANE (2016) y Ramírez, Zambrano, Mogrovejo y Carreño (2016), fueron empalmados anualmente con el fin de obtener un proxy de las estadísticas para el departamento.

En el horizonte indicado, este texto aborda inicialmente un breve marco conceptual sobre autonomía económica. En segundo lugar, se realiza un recuento normativo de las principales leyes que hablan sobre equidad de género en Colombia. Posteriormente, se describen las principales brechas de género en torno al mercado de trabajo; luego se realiza una discusión entre la evidencia empírica, el marco conceptual y las leyes, buscando con ello definir los esenciales retos del Estado en pro de la autonomía económica de las mujeres. Finalmente, se establecen algunas conclusiones del estudio.

\section{Autonomía económica de las mujeres y mercado de trabajo. Una aproxi- mación conceptual}

Teniendo en cuenta la Agenda 2030 y los Objetivos de Desarrollo Sostenible (CEPAL, 2016a), un aspecto importante para lograr la autonomía de las mujeres es "lograr el empleo pleno y productivo y el trabajo decente para todas las mujeres y los hombres, incluidos los jóvenes y las personas con discapacidad, así como la igualdad de remuneración por trabajo de igual valor"(CEPAL, 2016a, p.24). De este modo, analizar la autonomía económica de las mujeres requiere estudiar la distribución de los recursos económicos y productivos, entre hombres y mujeres, dentro de una sociedad.

Durante los últimos años, la autonomía económica se ha convertido en un tema relevante para la academia, en la medida en que investigadores sociales, gobiernos y organizaciones no gubernamentales (ONG), relacionan este concepto con estrategias que permitirán la reivindicación de los derechos de la mujer. Así, Tzannatos 
(1999), Deshmukh-Ranadive (2005), Oppenheim (2005), Petesch, Smulovitz, y Walton (2005), Addabbo, Arrizabalaga, Borderías, y Owens (2010), entre otros, establecen la autonomía económica como un proceso que posibilita a las mujeres convertirse en actoras sociales y políticas, adquiriendo un mayor poder económico dentro del hogar, un mayor poder de negociación en la familia.

Por su parte, Deere y León (2002), Deere (2012), Deere, Oduro, Swaminathan, y Doss (2013), Malapit y Quisumbing (2015), Akter, y otros (2017), piensan que la autonomía económica esta relacionada con el poder de decisión frente a los factores de producción, por ejemplo, en el caso de la mujer rural, las decisiones sobre la tierra son aspectos que miden el empoderamiento y poder de la mujer.

Así, la evidencia empírica demuestra que en aquellos países donde se aprecia un aumento en los niveles de autonomía económica también se perciben menores tasas de fecundidad (Dyson \& Moore, 1983; Mason, 1984; Schultz, 1990; Eswaran, 2002; Lupica \& Cogliandro, 2013), existen mayores niveles de educación (Quisumbing \& de la Briere, 2000; Pérez, 2006; Kabeer, 2009; Espino \& Sauval, 2016), los hogares perciben mayores ingresos (Blinder, 1973; Hoddinott \& Haddad, 1995; Thomas \& Frankenberg, 1997; Aspiazu, 2014) y reflejan un mayor desarrollo socioeconómico (García, 1989; UNDP, 1995; Benería, 2003; Halperín, 2011). Luego, la participación en el mercado de trabajo se convierte en un pilar fundamental para lograr la autonomía económica, pues el tener un empleo significa tener un ingreso, lo que aumenta el poder y la autonomía de la mujer (Golla, Malhotra, Nanda, \& Mehra, 2011). Según la CEPAL (2016),

La autonomía económica es un pilar fundamental de la autonomía de las mujeres y, por definición, requiere que estas perciban ingresos que les permitan superar la pobreza y disponer de su tiempo libremente para capacitarse, acceder al mercado laboral, desarrollarse profesional y personalmente. (p.39)

Así, las mujeres que no poseen un empleo tendrán menos dinero, afectando su acceso y control sobre los recursos productivos y educativos; de modo que el nulo, 0 precario empleo, limita su proceso de autonomía restringiendo su desarrollo dentro de la sociedad. 
Para Kabeer (2012), la autonomía económica, ligada al proceso de empoderamiento, se encuentra constreñida por dos tipos de limitaciones: las intrínsecas (reglas, creencias y costumbres) y las impuestas (donde el Estado y el mercado reproducen los estereotipos de género). Según esta autora, este tipo de restricciones definirán las preferencias de la demanda laboral, reproduciendo procesos de discriminación laboral y segregación ocupacional. Respecto a esteúltimo, Anker(2000)establece que la segregación ocupacional se encuentra dividida en dos: la segregación horizontal y la segregación vertical. Mientras la primera hace referencia a las diferencias en el acceso laboral que tienen las mujeres a algunas actividades económicas; la segunda se expresa en las diferencias de acceso a unos cargos u ocupaciones.

De este modo, la autonomía económica dependerá de varios aspectos: 1. La decisión de acceso al mercado de trabajo, 2. El acceso a trabajos no precarios, 3. El acceso a activos económicos, y 4 . La distribución equitativa de los recursos dentro del hogar (Calcagni \& Cortínez, 2017). Así, para estudiar la autonomía económica, Kabeer (2012) sugiere: 1. Estudiar la participación de la mujer al mercado de trabajo, 2. Analizar las características ocupacionales y trayectorias ocupacionales, y 3 . Definir las diferencias sexuales en términos monetarios.

Según Marco (2015), la autonomía económica se encuentra relacionada con la discriminación laboral de género. De este modo, la autonomía económica busca que las mujeres puedan "competir en los mercados en igualdad de condiciones con los hombres" (Marco, 2015, p.15). Para Deere y León (2002), Bennet (2002), Grown, Geeta, y Khan (2003), Pérez (2005), Kabber (2008), Espino (2012), entre otros, la autonomía económica apalanca la posibilidad de superar las restricciones y revertir las desigualdades de género dentro del mercado.

Cabe afirmar que para León (2001), la autonomía económica permite a la mujer acceder y controlar recursos, logrando con ello un empoderamiento que posibilitará redefinir las estructuras sociales que históricamente han reproducido las ideas preconcebidas sobre masculinidad y feminidad.

Así, la autonomía económica se constituye en un proceso de cambio en el que las mujeres asumen una mayor capacidad de toma de decisión y transformación frente a una sociedad excluyente (Sanchís \& Katzkowicz, 2014). De esta forma, el empoderamiento y la autonomía económica de las mujeres están relacionados con: 1. El acceso 
y control a recursos que le permitan competir en mercados, accediendo de forma equitativa, justa, sin barreras institucionales, posibilitándole avanzar económicamente. 2. La posibilidad de participar en procesos de toma de decisiones, acrecentando su poder y respetando su agencia (Golla, et al., 2011).

\section{Marco jurídico para la equidad de género de las mujeres en Colombia. Un estado del arte}

Colombia se caracteriza por su excesivo apego a las leyes, así estas se expidan al margen de los conflictos que pretende solucionar o no afecten la realidad. Este fetichismo normativo explica por qué la respuesta inmediata frente a un problema social, político o económico es la expedición de una norma, la cual fácilmente cae en el olvido hasta que una nueva urgencia conlleve a una nueva expedición normativa. Así, por ejemplo, la necesidad de cumplir con estándares internacionales en materia de igualdad entre hombres y mujeres, permitió la expedición de la Ley 51 de 1981, en virtud de la cual Colombia ratificó la Convención sobre la eliminación de todas las formas de discriminación contra las mujeres (CEDAW, por sus siglas en inglés). Sin embargo, hasta 1991, estos tratados internacionales no reglamentados carecían de toda exigibilidad interna, razón por la cual, dicha norma, a pesar de su enorme importancia en la consecución de la autonomía femenina, al comprometer a los Estados con la eliminación de toda clase de discriminación basada en el sexo, pasó casi que inadvertida para la sociedad colombiana e inclusive para las propias mujeres y sus organizaciones.

La convocatoria de la Asamblea Nacional Constituyente de 1990 y la presencia activa de las mujeres pertenecientes a grupos políticos como la Unión Patriótica (Aida Abello), al M-19 (Maria Mercedes Carranza y María Teresa Garcés) y el Partido Liberal (Helena Herrán), y que hicieron suyas las reclamaciones planteadas por 17 organizaciones de mujeres, permitieron el logro de reivindicaciones que se sintetizan en la constitucionalización de la Convención para la eliminación de todas las formas de discriminación contra la mujeres (CEDAW). Estos postulados se positivizaron para eliminar las brechas laborales, políticas, familiares, educativas y sociales entre hombres y mujeres, a fin de superar la situación de discriminación sistemática e 
histórica que padecía la mitad de la población, por la sola razón de pertenecer al sexo femenino.

Así, el Estado Social de Derecho construido sobre las bases del nuevo constitucionalismo, comprometido con la justicia material y la igualdad real, reconoció la pluralidad política y la multiculturalidad étnica como rasgos identitarios de la sociedad colombiana. Luego consagró una Carta de Derechos con acciones constitucionales sobre las cuales debía reconstruirse la administración de justicia, atada al respeto a la dignidad humana, la igualdad de oportunidades, la tolerancia, el trabajo y la paz, principios que orientan el ordenamiento jurídico colombiano y que se encuentran en el preámbulo, no como mera retórica sino con fuerza vinculante, tal como lo definió la jurisprudencia constitucional.

En torno a las conquistas constitucionales que benefician no solo a las mujeres sino a toda la población trabajadora, se encuentran los artículos 25 y 26 que consagran la protección especial que merece el trabajo en cualquier modalidad que se presente y la libertad de escoger profesión y oficio, con la sola restricción de la idoneidad y la responsabilidad social.

Por su parte, el artículo 43, quizá el de mayor importancia en relación con el tema de investigación, consagra que:

La mujer y el hombre tienen iguales derechos y oportunidades. La mujer no podrá ser sometida a ninguna clase de discriminación. Durante el embarazo y después del parto gozará de especial asistencia y protección del Estado, y recibirá de éste subsidio alimentario si entonces estuviere desempleada o desamparada. El Estado apoyará de manera especial a la mujer cabeza de familia.

Así, bajo el escenario anterior, se configuraron las bases para el empoderamiento de derechos y la construcción de la autonomía económica de las mujeres. De esta forma, todas estas disposiciones consagran derechos y estrategias que buscan que puedan realizarse como iguales en una sociedad tradicionalmente discriminadora. Pasan de ser sujetos de protección a sujetos titulares de derechos con autonomía y poder decisorio, armonizando el derecho interno con los derechos que ya habían sido reconocidos en tratados internacionales. La figura del bloque de constituciona- 
lidad consagrado en el Art. 93 engrosó los derechos consagrados en la Constitución, con aquellos contenidos en los tratados internacionales de derechos humanos y en especial con la Convención para la eliminación de todas las formas de discriminación contra la mujer (CEDAW), ratificada en Colombia por medio de la Ley 51 de 1981 y, después de la Constitución de 1991, reconocidos a partir de diferentes disposiciones y normas sobre igualdad de género (Goyes, 2011).

Sobre este último aspecto, frente a las principales normas que regulan la equidad de género se encuentran:

La Ley 82 de 1993, que le confiere protección especial a la mujer cabeza de familia y que posteriormente se hizo extensiva a los hombres cabeza de familia. La Ley 248 de 1995, que ratificó la Convención de Belén Do Pará para prevenir, sancionar y erradicar todas las formas de violencia contra la mujer. La Ley 188 de 1996, por medio de la cual se creó la Dirección nacional para la equidad de género. La Ley 278 de 1996, sobre la Comisión permanente de concertación de políticas salariales y laborales creada por el artículo 56 de la Constitución Política. El Decreto 1182 de 1999, que se ocupó de modificar la Dirección en Alta Consejería Presidencial para la equidad de la mujer. La Ley 581 de 2000 o Ley de cuotas, tendiente a garantizar la vinculación de la mujer en al menos el 30\% de los cargos de dirección del Estado. La Ley 640 de 2001, sobre conciliación en asuntos de familia. La Ley 731 de 2002, sobre las normas para favorecer a las mujeres rurales. La Ley 823 de 2003, por la que se dictan normas sobre igualdad de oportunidades para las mujeres. La Ley 1009 de 2006, en virtud de la cual se creó el Observatorio de Asuntos de Género. La Ley 1010 de 2006, que reguló el acoso laboral y el acoso sexual. La Ley 1232 de 2008, que se encargó de redefinir la Jefatura de hogar para incluir a la mujer. La Ley 1257 de 2008, sobre la no violencia contra las mujeres, busca reconocer social y económicamente los derechos de las mujeres. La Ley 1413 de 2010, regula la inclusión de la economía del cuidado en el sistema de cuentas nacionales. El Decreto 164 de 2010, creó la Mesa interinstitu- 
cional para erradicar la Violencia contra la mujer. La Ley 1429 de 2010, sobre la ley de formalización y generación de empleo. El Decreto 4463 de 2011, sobre la equidad salarial entre hombres y mujeres. El Decreto 4796 de 2011, que reglamentó los artículos 8, 9, 13 y 19 de la Ley 1257 de 2008, para garantizar el derecho a la salud de las mujeres víctimas de violencia de género, obligando a otorgar atención integral. La Ley 1434 de 2011, crea la comisión para la equidad de la mujer del Congreso de la República. Ley 1468 de 2011, que elevó a 14 semanas la licencia de maternidad. La Ley 1496 de 2011, sobre la igualdad salarial y de la retribución laboral entre mujeres y hombres. El Decreto 4798 de 2011, que se ocupó de la adopción de medidas de prevención de la violencia basada en género en el ámbito educativo. El Decreto 4799 de 2011, sobre las competencias de las Comisarías de familia, Fiscalía General de la Nación, juzgados Civiles y de Control de Garantías para un efectivo y pronto acceso a la justicia. El Decreto 2734 de 2012, sobre la atención a las víctimas de violencia. El Decreto 2733 de 2012, que reguló deducciones sobre la renta a favor de aquellos empresarios que vinculen laboralmente a mujeres víctimas de violencia basada en género. La Ley 1542 de 2012, que se ocupó de la violencia intrafamiliar y la inasistencia alimentaria, tipificados en los artículos 229 y 233 del Código Penal. La Ley 1652 de 2013, que estableció pautas para realizar entrevistas a niñas y niños víctimas de delitos contra la libertad sexual. El Decreto 1930 de 2013, por el que se adopta la Política Pública Nacional de Equidad de Género. La Ley 1719 de 2014 que estableció medidas para permitir el acceso a la justicia de las víctimas de violencia sexual, en especial los casos asociados al conflicto armado. La Ley 1761 de 2015, que consagró el feminicidio como un tipo penal autónomo. La Ley 1773 de 2016, relativa a las víctimas de ataques con ácido, y la Ley 1857 de 2017, sobre medidas de protección a la familia, flexibilización de la jornada laboral y la celebración del día de la familia. (Goyes, 2011) 
Del anterior listado y por su incidencia directa para alcanzar la autonomía económica de la mujer, se hará especial referencia a la Ley 82 de 1993, por medio de la cual se dictan normas para apoyar de manera directa a la mujer cabeza de familia. La Ley 731 de 2002 es otra medida legislativa para garantizar la protección de la mujer rural, al dar un tratamiento especial a mujeres de estos sectores de la sociedad tradicionalmente, olvidadas y discriminadas, incluyendo medidas que permitan alcanzar la igualdad formal y real. Al respecto se planteó la creación del Fondo de Fomento para las Mujeres Rurales (Fommur) del Ministerio de Agricultura, con la finalidad de apoyar los planes, programas y proyectos que lleven a la incorporación de las mujeres rurales dentro de la vida política, económica y social. Sin embargo, no existe un decreto que materialice lo descrito en la Ley, y las directrices de las políticas públicas no tienen suficiente claridad para contribuir al mejoramiento de la calidad de vida de estas mujeres que tienen una participación importante en la economía campesina.

La Ley 823 de 2003 estableció normas sobre la igualdad de oportunidades para las mujeres en el ámbito público y privado. Esta ley establece un marco institucional para orientar las políticas y acciones que garanticen la equidad y la igualdad a fin de que las mujeres puedan ejercer plenamente sus derechos políticos, civiles, económicos, sociales y culturales, permitiendo así, el desarrollo de sus aptitudes y capacidades y la participación en los diferentes campos de la vida nacional. Para esto se deben eliminar los obstáculos que impiden ese pleno ejercicio de sus derechos, incorporando políticas y acciones de equidad de género e igualdad de oportunidades.

Desde el año 2010 se expidió la Ley 1413 que reguló la inclusión económica de las actividades de cuidado en el Sistema de Cuentas Nacionales, con el objeto de medir la contribución de la mujer al desarrollo económico y social, facilitando su implementación en las políticas públicas. Esta Ley logró un reconocimiento de las labores de cuidado como una actividad productiva que favorece la economía del país, además busca la redistribución de las responsabilidades del cuidado entre hombres y mujeres, eliminación de sobrecarga de las jornadas en las mujeres, circunstancia que atenta contra su salud física y psíquica. 
Por su parte, la Ley 1496 de 2011 busca hacer efectivo el derecho a la igualdad salarial, promoviendo el ingreso de las mujeres a espacios productivos no tradicionales para las mujeres.

En el mismo sentido, la Ley 1857 de 2017 adoptó una serie de medidas tendientes a garantizar la armonización de la vida laboral con la vida familiar, generando instrumentos especiales, tales como, la flexibilización de la jornada laboral, la obligación para empleadores públicos como privados, de poner en marcha la celebración del día de la familia como una actividad que permita vincular estos dos espacios de la vida personal, lo familiar y lo laboral.

Cabe recordar que el Plan Nacional de Desarrollo 2010-2014 contiene la política pública nacional integral de equidad de género para las mujeres. En septiembre de 2012 el gobierno lanzó los lineamientos de esta política que se constituyó en un referente para el diseño de las acciones sostenibles encaminadas a la transformación y superación de las brechas de desigualdad y el goce efectivo de los derechos de las mujeres. Con fundamento en esos lineamientos se construyó, en el año 2013, el documento Conpes 161 , sobre los lineamientos para la política pública nacional de equidad de género para las mujeres. En este documento se definen seis ejes: 1) Construcción de paz y transformación cultural, 2) Autonomía económica y acceso a activos, 3) Participación en los escenarios de poder y de toma de decisión, 4) Salud y derechos sexuales y reproductivos, 5) Enfoque de género en la educación, y 6) Plan integral para garantizar a las mujeres una vida libre de violencias.

Finalmente, en la Ley 1753 de 2015, por la que se expide el Plan Nacional de Desarrollo 2014-2018, en los Artículos 107, 129 y 232, se expresan los diferentes compromisos sobre la mujer. Específicamente, en el Artículo 107 se busca crear la Dirección de Mujer Rural que se encargará de desarrollar la Política Pública Integral de Mujer Rural. Por su parte, el Artículo 129 pretende impulsar una evaluación participativa de la Política Pública Nacional de Equidad de Género para las Mujeres y de la Política Pública para la Prevención de Riesgos, la Protección y Garantía de los Derechos de las Mujeres Víctimas del conflicto armado; mientras que el Artículo 232, sobre la política pública de la mujer rural, busca que el gobierno formule una política integral con enfoque étnico, etario y territorial, buscando superar las brechas urbano-rurales. 


\section{Mercado de trabajo. Brechas de género y determinantes del ingreso laboral}

\section{Principales brechas sexuales en el mercado de trabajo de Nariño}

Durante los últimos cinco años, el promedio de la brecha en la tasa global de participación del departamento de Nariño se ha mantenido cercana al 18,6\%. No obstante, la participación de la mujer en el mercado de trabajo, al igual que la de los hombres, creció en este periodo, al pasar de $58,56 \%$ a $61,2 \%$ (Tabla 1).

Tabla 1. Nariño. Tasa Global de Participación. 2013 a 2017-I'

\begin{tabular}{|c|c|c|c|c|}
\hline PERIODO & TOTAL & HOMBRE & MUJER & BRECHA \\
\hline 2013 & $67,61 \%$ & $77,27 \%$ & $58,56 \%$ & $18,71 \%$ \\
\hline 2014 & $67,60 \%$ & $76,61 \%$ & $58,14 \%$ & $18,47 \%$ \\
\hline 2015 & $68,32 \%$ & $77,38 \%$ & $58,56 \%$ & $18,82 \%$ \\
\hline 2016 & $69,39 \%$ & $78,47 \%$ & $59,72 \%$ & $18,75 \%$ \\
\hline $2017-I$ & $70,25 \%$ & $79,93 \%$ & $61,20 \%$ & $18,73 \%$ \\
\hline
\end{tabular}

Fuente: Cálculos del Observatorio de Género de Nariño a partir de GEIH - DANE.

De acuerdo con algunos autores como García (2001), Galvis (2010), Gasparini y Marchionni (2015), Arango y Ríos (2015) y Ramoni y Orlandoni (2017), el crecimiento de TGP de la mujer se relaciona con la mayor salida de mujeres jóvenes al mercado de trabajo, buscando con ello aumentar los ingresos de los hogares que actualmente ya no dependen de un perceptor sino de varios.

Por su parte, la tasa de ocupación, durante los años 2013-2017-I presenta una reducción de la brecha de género, la cual pasó de 22,59 \% a 22,09\% (Tabla 2). A pesar de ello, este indicador es mayor para los hombres que para las mujeres. De acuerdo a Campaña, Giménez-Nadal y Molina (2018) y Krinickiene (2018), esta diferencia en el indicador puede ser causada por factores relacionados con discriminación laboral. 
Tabla 2. Nariño. Tasa de ocupación. 2013 a 2017-।

\begin{tabular}{|c|c|c|c|c|}
\hline PERIODO & TOTAL & HOMBRE & MUJER & BRECHA \\
\hline 2013 & $60,33 \%$ & $72,85 \%$ & $50,26 \%$ & $22,59 \%$ \\
\hline 2014 & $60,88 \%$ & $72,66 \%$ & $49,75 \%$ & $22,90 \%$ \\
\hline 2015 & $62,11 \%$ & $74,85 \%$ & $51,17 \%$ & $23,67 \%$ \\
\hline 2016 & $64,12 \%$ & $74,47 \%$ & $53,42 \%$ & $21,06 \%$ \\
\hline $2017-I$ & $65,70 \%$ & $77,11 \%$ & $55,02 \%$ & $22,09 \%$ \\
\hline
\end{tabular}

Fuente: Cálculos del Observatorio de Género de Nariño a partir de GEIH - DANE.

No obstante, para el caso de Nariño, gran parte de estas diferencias se encuentran explicadas, como lo señalan Sarmiento y Vargas (2002), por la “invisibilización” en las estadísticas del trabajo de la mujer campesina, que en el caso del departamento se hacen muy presentes. Sobre este tema, según Díaz (2002), en Colombia, estadística y económicamente, se ha negado la existencia del trabajo femenino, dando paso a una visión dualista de la economía: "la economía de los bienes" y "la economía oculta", donde:

(...) la economía de los bienes, (es) la que se considera 'la economía propiamente dicha'; y por otro lado, la economía oculta, invisible, la economía del cuidado. Lo que las diferencia es que el trabajo en la segunda no es remunerado, no se contabiliza y sobre todo es realizado principalmente por las mujeres, sin distinción de edad, raza o etnia. (Campillo, 1999)

Luego, es claro que gran parte de las mujeres que se encuentren trabajando en zonas rurales no aparezcan en las estadísticas de ocupación, sobre todo cuando sus labores son naturalizadas al cuidado familiar y no son reconocidas como un trabajo.

Con respecto a la tasa de desempleo para Nariño, la brecha de género, durante los años 2013-2017-I, se ubicó en promedio cercana al $7 \%$ a favor de los hombres (Tabla 3). No obstante, desde el año 2013 hasta el año 2015 se observa una tendencia creciente, posteriormente la brecha se reduce. De acuerdo con la CEPAL (2017) esta tendencia, en Latinoamérica, está relacionada en gran parte con la estructura social que reproduce estereotipos, limitando la inserción de la mujer a diferentes 
ocupaciones. Según Arriagada (2006), las mujeres son más susceptibles a sufrir el desempleo, mientras que los hombres son más propensos a estar en la informalidad.

Tabla 3. Nariño. Tasa de desempleo. 2013 a 2017-I

\begin{tabular}{|c|c|c|c|c|}
\hline PERIODO & TOTAL & HOMBRE & MUJER & BRECHA \\
\hline 2013 & $11,06 \%$ & $7,64 \%$ & $15,44 \%$ & $-7,80 \%$ \\
\hline 2014 & $10,53 \%$ & $7,00 \%$ & $15,59 \%$ & $-8,59 \%$ \\
\hline 2015 & $9,20 \%$ & $5,39 \%$ & $14,20 \%$ & $-8,81 \%$ \\
\hline 2016 & $7,91 \%$ & $4,70 \%$ & $11,68 \%$ & $-6,98 \%$ \\
\hline $2017-1$ & $7,63 \%$ & $4,86 \%$ & $11,02 \%$ & $-6,16 \%$ \\
\hline
\end{tabular}

Fuente: Cálculos del Observatorio de Género de Nariño a partir de GEIH - DANE.

Así, confirmando la hipótesis de Arriagada (2006), se encuentra que en Nariño los hombres son más susceptibles a emplearse bajo condiciones informales que las mujeres (Tabla 4); sin embargo, la brecha sexual en este indicador es muy pequeño, de ahí que se pueda concluir que la informalidad², por lo menos en el departamento analizado, es un problema que afecta de igual forma tanto a hombres como a mujeres.

Tabla 4. Nariño. Informalidad laboral. 2013 a 2017-।

\begin{tabular}{|c|c|c|c|c|}
\hline PERIODO & TOTAL & HOMBRE & MUJER & BRECHA \\
\hline 2013 & $78,03 \%$ & $79,11 \%$ & $76,53 \%$ & $2,57 \%$ \\
\hline 2014 & $77,63 \%$ & $78,96 \%$ & $75,79 \%$ & $3,17 \%$ \\
\hline 2015 & $77,83 \%$ & $79,45 \%$ & $75,55 \%$ & $3,90 \%$ \\
\hline 2016 & $79,45 \%$ & $81,19 \%$ & $77,14 \%$ & $4,05 \%$ \\
\hline $2017-I$ & $77,53 \%$ & $78,34 \%$ & $76,48 \%$ & $1,86 \%$ \\
\hline
\end{tabular}

Fuente: Cálculos del Observatorio de Género de Nariño a partir de GEIH - DANE.

2 De acuerdo con el DANE (2009), se pueden categorizar como informales a: "1. Los empleados particulares y los obreros que laboran en establecimientos, negocios o empresas que ocupen hasta cinco personas en todas sus agencias y sucursales, incluyendo al patrono y/o socio; 2 . Los trabajadores familiares sin remuneración; 3. Los trabajadores sin remuneración en empresas o negocios de otros hogares; 4. Los empleados domésticos; 5. Los jornaleros o peones; 6 . Los trabajadores por cuenta propia que laboran en establecimientos hasta cinco personas, excepto los independientes profesionales; 7. Los patrones o empleadores en empresas de cinco trabajadores o menos; 8 . Se excluyen los obreros o empleados del gobierno". 
De acuerdo con Galvis-Aponte y Pérez-Valbuena (2015), las altas tasas de informalidad observadas en Nariño pueden ser explicadas por: 1. La existencia de un alto porcentaje de población trabajadora que no posee un nivel educativo superior a la secundaria, población que estará más vulnerable a incluirse en empleos de baja calidad; y 2. El entorno económico de Nariño es dependiente del sector terciario, especialmente en actividades de comercio y servicios, definido por las microempresas, donde los trabajadores son empleados como asalariados en empleos precarios y en muchas ocasiones ejercen como cuenta propia. Sobre este último aspecto, Weller (2004) afirma que en Latinoamérica se vivió un proceso de terciarización espuria, especialmente desde la década de los ochenta cuando se percibe un proceso de "sobreterciarización" donde el empleo estaba concentrado en el sector terciario o sectores de baja productividad.

(...) algunos de los servicios tienen las barreras de entrada más bajas de todas las ramas de actividad, debido a bajos o nulos requisitos de capital, tierra, tecnología y capital humano, por lo que pueden servir de "refugio" a una fuerza laboral que no encuentra empleo en actividades más productivas y mejor remuneradas. De esta manera, el peso de las actividades informales suele ser significativamente más elevado en el sector terciario que en el secundario. (Weller, 2004, pp.162-163)

Así, en el caso de Nariño, la terciarización del empleo, especialmente en las mujeres, está determinado por las ventas ambulantes, salones de belleza, ventas por catálogo, ventas de comidas rápidas, entre otras actividades que no generan ingresos suficientes para lograr un nivel de vida digno. Luego, no es extraño observar que, durante el periodo de estudio, la actividad de comercio, restaurantes y hoteles; al igual que las actividades de establecimientos financieros y servicios sociales y comunales se encuentran feminizadas (Tabla 5).

De igual forma, la terciarización y su tendencia a la informalidad y el empleo precario se hace más evidente en el análisis por oficio, donde se observa una alta feminización en las ocupaciones de comerciantes y vendedores, y trabajadores en servicios (Tabla 6). Sobre este aspecto, siguiendo la tesis de Weller (2004), gran parte de este segmento de trabajadores se caracteriza por un bajo nivel educativo y la pobreza. 
Tabla 5. Nariño. Índice de feminización por actividad económica. 2013 a 2017-I

\begin{tabular}{|l|l|l|l|l|l|l|}
\hline \multicolumn{1}{|c|}{ ACTIVIDAD ECONÓMICA } & $\mathbf{2 0 1 3}$ & $\mathbf{2 0 1 4}$ & $\mathbf{2 0 1 5}$ & $\mathbf{2 0 1 6}$ & $\mathbf{2 0 1 7 - I}$ & \multicolumn{1}{c|}{ CARACTERISTICA } \\
\hline Agricultura, silvicultura, caza y pesca & 0,35 & 0,39 & 0,36 & 0,39 & 0,50 & Infrarepresentación \\
\hline Explotación de minas y canteras & 0,11 & 0,31 & 0,12 & 0,04 & 0,15 & Infrarepresentación \\
\hline Industrias manufactureras & 1,07 & 1,02 & 0,96 & 0,97 & 0,67 & Infrarepresentación \\
\hline Electricidad, gas y agua & 0,53 & 0,15 & 0,12 & 0,56 & 0,25 & Infrarepresentación \\
\hline Construcción & 0,05 & 0,05 & 0,05 & 0,04 & 0,03 & Infrarepresentación \\
\hline Comercio, restaurantes y hoteles & 1,42 & 1,46 & 1,48 & 1,57 & 1,48 & Feminización \\
\hline Transporte y almacenamiento & 0,23 & 0,17 & 0,12 & 0,14 & 0,12 & Infrarepresentación \\
\hline Establecimientos financieros & 1,14 & 1,30 & 1,22 & 1,35 & 1,25 & Feminización \\
\hline Servicios sociales y comunales & 2,12 & 2,06 & 2,10 & 2,22 & 2,09 & Feminización \\
\hline Total & 0,72 & 0,72 & 0,71 & 0,76 & 0,77 & Infrarepresentación \\
\hline
\end{tabular}

Fuente: Cálculos del Observatorio de Género de Nariño a partir de GEIH - DANE.

Tabla 6. Nariño. Índice de feminización por oficio principal. 2013 a 2017-I

\begin{tabular}{|l|l|l|l|l|l|l|}
\hline \multicolumn{1}{|c|}{ OFICIO PRINCIPAL } & $\mathbf{2 0 1 3}$ & $\mathbf{2 0 1 4}$ & $\mathbf{2 0 1 5}$ & $\mathbf{2 0 1 6}$ & $\mathbf{2 0 1 7 - I}$ & CARACTERISTICA \\
\hline Director o funcionario superior & 0,83 & 0,70 & 0,69 & 0,65 & 0,53 & Infrarepresentación \\
\hline Administrativo y nivel intermedio & $\mathbf{1 , 6 1}$ & 1,51 & 1,54 & 1,72 & 1,37 & Feminización \\
\hline Profesional y técnico & 0,97 & 0,88 & 0,98 & 1,07 & 1,05 & Equidad \\
\hline Comerciantes y vendedores & 1,65 & 1,77 & 1,72 & 1,72 & 1,61 & Feminización \\
\hline Trabajadores en servicios & 3,83 & 3,81 & 3,32 & 4,01 & 3,12 & Feminización \\
\hline Trabajadores agrícolas & 0,34 & 0,39 & 0,36 & 0,39 & 0,49 & Infrarepresentación \\
\hline $\begin{array}{l}\text { Obreros no agrícolas, conductores de } \\
\text { maquinaria y servicios de transporte }\end{array}$ & 0,27 & 0,26 & 0,27 & 0,25 & 0,20 & Infrarepresentación \\
\hline Otras & 0,26 & 0,33 & 0,35 & 0,56 & 0,36 & Infrarepresentación \\
\hline Total & 0,72 & 0,72 & 0,71 & 0,76 & 0,77 & Infrarepresentación \\
\hline
\end{tabular}

Fuente: Cálculos del Observatorio de Género de Nariño a partir de GEIH - DANE.

En general, las tablas 5 y 6 también posibilitaron observar que en Nariño existen actividades o cargos "típicamente" masculinos que definen barreras que impiden la entrada de las mujeres. Sobre ello, Anker (2000) afirma que los procesos de segregación ocupacional o discriminación laboral dan paso a las expresiones de "techo de cristal" o "suelo pegajoso". 


\section{Estimaciones de la brecha salarial de Nariño}

En cuanto al ingreso familiar por sexo se encuentra que los hogares que tienen como jefe a una mujer, durante el periodo analizado, presentaron en promedio un ingreso menor en $5,2 \%$. Sobre esto, si bien el ingreso monetario en términos corrientes se encuentra aumentando, aún los ingresos de los hombres son mayores que lo de las mujeres (Tabla 7).

Tabla 7. Nariño. Promedio ingreso familiar por sexo del jefe de hogar. 2013 a 2017-I

\begin{tabular}{|c|c|c|c|c|c|}
\hline PERIODO & TOTAL & HOMBRE & MUJER & BRECHA & BRECHA (\%) \\
\hline 2013 & $\$ 779.377$ & $\$ 807.153$ & $\$ 752.608$ & $\$ 54.545$ & $6,76 \%$ \\
\hline 2014 & $\$ 851.998$ & $\$ 865.234$ & $\$ 839.398$ & $\$ 25.836$ & $2,99 \%$ \\
\hline 2015 & $\$ 930.062$ & $\$ 964.377$ & $\$ 896.703$ & $\$ 67.674$ & $7,02 \%$ \\
\hline 2016 & $\$ 865.691$ & $\$ 886.011$ & $\$ 846.401$ & $\$ 39.610$ & $4,47 \%$ \\
\hline $2017-I$ & $\$ 1.120 .804$ & $\$ 1.149 .526$ & $\$ 1.093 .863$ & $\$ 55.663$ & $4,84 \%$ \\
\hline
\end{tabular}

Fuente: Cálculos del Observatorio de Género de Nariño a partir de GEIH - DANE.

Un comportamiento parecido al anterior se observa en la diferencia de medias del ingreso laboral por sexo (Tabla 8). Respecto a este análisis se puede afirmar que la brecha de salario a favor de los hombres se encuentra cercana a 13,7 \% para el periodo analizado. De acuerdo con Ñopo (2012) las brechas salariales en Colombia no solo se pueden explicar por la diferencia en educación y las características demográficas, sino también por las características ocupacionales y el tamaño de las empresas. En este sentido, para Nariño las brechas se relacionan con la terciarización y el predominio del sector microempresarial.

Tabla 8. Nariño. Promedio ingreso laboral por sexo. 2013 a 2017-I

\begin{tabular}{|c|c|c|c|c|c|}
\hline PERIODO & TOTAL & HOMBRE & MUJER & BRECHA & BRECHA (\%) \\
\hline 2013 & $\$ 722.446$ & $\$ 775.251$ & $\$ 665.749$ & $\$ 109.502$ & $14,12 \%$ \\
\hline 2014 & $\$ 752.788$ & $\$ 821.854$ & $\$ 686.109$ & $\$ 135.745$ & $16,52 \%$ \\
\hline 2015 & $\$ 786.691$ & $\$ 839.798$ & $\$ 732.513$ & $\$ 107.285$ & $12,78 \%$ \\
\hline 2016 & $\$ 787.407$ & $\$ 831.293$ & $\$ 746.039$ & $\$ 85.253$ & $10,26 \%$ \\
\hline $2017-1$ & $\$ 929.424$ & $\$ 1.002 .755$ & $\$ 852.937$ & $\$ 149.818$ & $14,94 \%$ \\
\hline
\end{tabular}

Fuente: Cálculos del Observatorio de Género de Nariño a partir de GEIH - DANE. 
Por otra parte, un análisis de la densidad de los ingresos laborales deflactados de acuerdo al índice de precios al consumidor de cada año, revela que el salario real, entre los años 2013-2016, tanto para hombres como para mujeres, se ha incrementado. No obstante, la brecha del ingreso por hora es persistente (Figura 1).
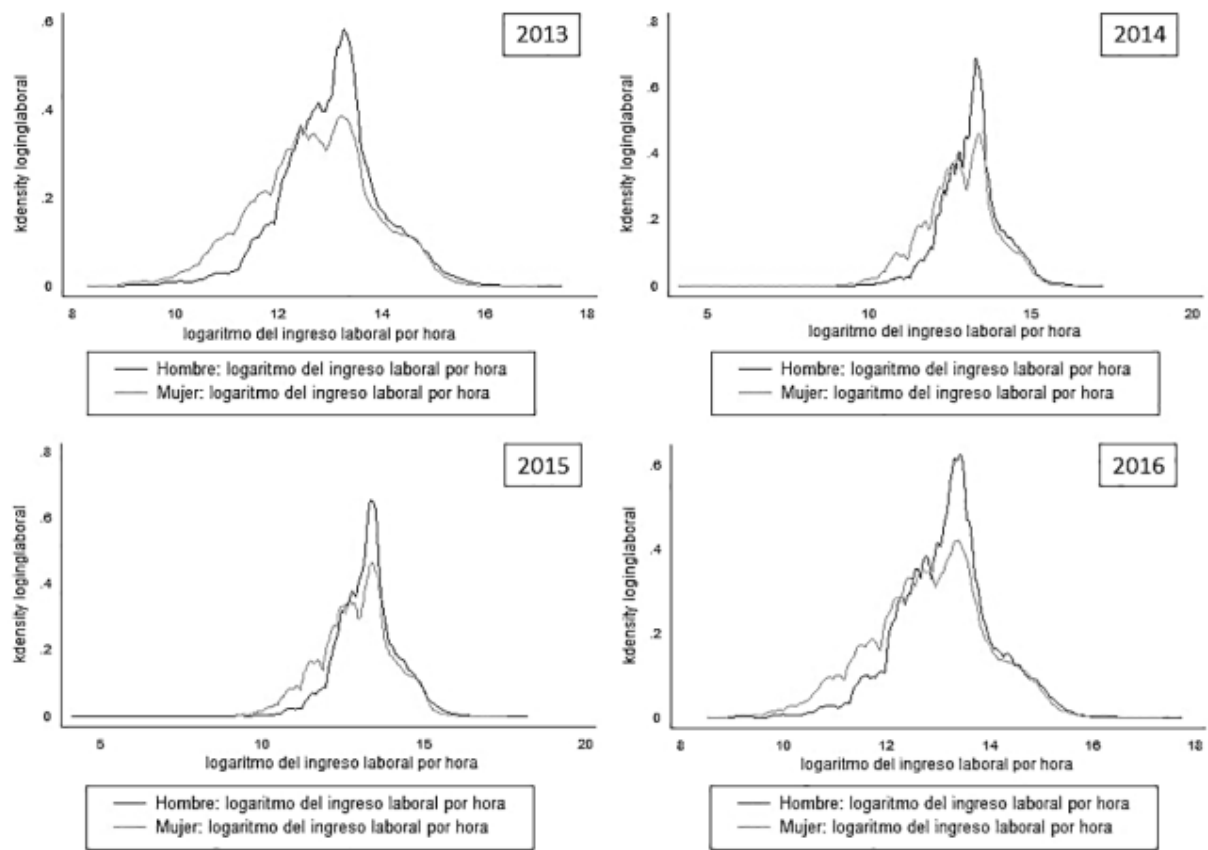

Figura 1. Nariño. Densidad de los salarios de hombres y mujeres. 2013-2016. Fuente: Cálculos del Observatorio de Género de Nariño a partir de GEIH - DANE.

Luego, como lo muestra la Tabla 9, las mujeres de Nariño reciben en promedio $25,7 \%$ menos ingreso que los hombres, porcentaje muy similar al que se espera encontrar más adelante en el modelo de descomposición de Oaxaca-Blinder. Sobre este aspecto es relevante aclarar que la diferencia expresada anteriormente no manifiesta la existencia de discriminación, pues este coeficiente es el promedio de las diferencias en los niveles de educación y experiencia, entre mujeres y hombres. 
Tabla 9. Nariño. Estimación del ingreso laboral (logaritmo) por sexo

\begin{tabular}{|c|c|c|c|}
\hline VARIABLES & TOTAL & HOMBRE & MUJER \\
\hline \multicolumn{4}{|l|}{ LOGARITMO DEL INGRESO } \\
\hline \multirow{2}{*}{ Años de educación } & $0,151^{* * *}$ & 0,0988 & $0,163^{* * *}$ \\
\hline & $(0,0564)$ & $(0,130)$ & $(0,0605)$ \\
\hline \multirow{2}{*}{ Experiencia } & $0,0325^{* * *}$ & $0,0349 * * *$ & $0,0290 * * *$ \\
\hline & $(0,00466)$ & $(0,0103)$ & $(0,00523)$ \\
\hline \multirow{2}{*}{ Educación primaria } & $-0,187^{*}$ & 0,00600 & $-0,255^{*}$ \\
\hline & $(0,328)$ & $(0,827)$ & $(0,342)$ \\
\hline \multirow{2}{*}{ Educación secundaria } & $0,248^{*}$ & $0,463^{*}$ & $0,364^{*}$ \\
\hline & $(0,440)$ & $(1,054)$ & $(0,466)$ \\
\hline \multirow{2}{*}{ Educación superior } & $0,285^{*}$ & 0,0349 & $0,437^{*}$ \\
\hline & $(0,593)$ & $(1,400)$ & $(0,632)$ \\
\hline \multirow{2}{*}{ Sexo (1=Mujer) } & $-0,257^{* * *}$ & & \\
\hline & $(0,139)$ & & \\
\hline \multirow{2}{*}{ Constant } & $10,48^{* * *}$ & $10,50^{* * *}$ & $10,15^{* * *}$ \\
\hline & $(0,475)$ & $(0,999)$ & $(0,474)$ \\
\hline \multicolumn{4}{|c|}{ PEA (EFECTOS MARGINALES) } \\
\hline \multirow{2}{*}{ Edad } & $0,182^{* * *}$ & $0,186^{* * *}$ & $0,202^{* * *}$ \\
\hline & $(0,00992)$ & $(0,0183)$ & $(0,0129)$ \\
\hline \multirow{2}{*}{ Edad al cuadrado } & $-0,00228^{* * *}$ & $-0,00226^{* * *}$ & $-0,00257^{* * *}$ \\
\hline & $(0,000125)$ & $(0,000222)$ & $(0,000165)$ \\
\hline \multirow{2}{*}{ Familiares menores de 6 años } & $0,117^{* * *}$ & 0,0757 & $-0,0831^{*}$ \\
\hline & $(0,0444)$ & $(0,127)$ & $(0,0489)$ \\
\hline \multirow{2}{*}{ Familiares mayores de 60 años } & $0,119^{* * *}$ & 0,0456 & $0,0977^{*}$ \\
\hline & $(0,0420)$ & $(0,0819)$ & $(0,0515)$ \\
\hline \multirow{2}{*}{ Otros ingresos } & $-7,02 \mathrm{e}-08^{* * *}$ & $-1,37 e-08$ & $-1,02 \mathrm{e}-07^{* * *}$ \\
\hline & $(2,32 \mathrm{e}-08)$ & $(3,44 \mathrm{e}-08)$ & $(3,14 \mathrm{e}-08)$ \\
\hline \multirow{2}{*}{ Otros desempleados en el hogar } & 0,0757 & $0,218^{*}$ & $-0,00194$ \\
\hline & $(0,0663)$ & $(0,125)$ & $(0,0811)$ \\
\hline \multirow{2}{*}{ Compromiso (1=Si) } & $-0,0361$ & $0,349^{* *}$ & $-0,289^{* * *}$ \\
\hline & $(0,0597)$ & $(0,158)$ & $(0,0734)$ \\
\hline \multirow{2}{*}{ Jefe de hogar (1=Si) } & $0,596^{* * *}$ & $0,648^{* * *}$ & $0,374^{* * *}$ \\
\hline & $(0,0677)$ & $(0,165)$ & $(0,0843)$ \\
\hline \multirow{2}{*}{ Constant } & $-5,140 * * *$ & $-5,256^{* * *}$ & $-5,226 * * *$ \\
\hline & $(0,191)$ & $(0,353)$ & $(0,239)$ \\
\hline \multirow[t]{2}{*}{ Lambda } & $-0,245^{*}$ & $-0,154$ & $-0,261^{*}$ \\
\hline & $(0,144)$ & $(0,281)$ & $(0,156)$ \\
\hline Observations & 9,102 & 3,170 & 5,932 \\
\hline
\end{tabular}

Robust standard errors in parentheses

${ }^{* * *} p<0.01,{ }^{* *} p<0.05,{ }^{*} p<0.1$

Fuente: Cálculos del Observatorio de Género de Nariño a partir de GEIH - DANE. 
Asimismo, de lo anterior, se puede concluir que: 1. Los retornos por años de educación son mayores para mujeres que para hombres; 2 . Por cada año adicional de experiencia los hombres tienen un retorno sobre el ingreso cercano a 3,4\%, mientras que las mujeres solo reciben el $2,9 \% ; 3$. El contar solamente con educación primaria es una restricción para las mujeres para obtener un ingreso; sin embargo, a medida que se incrementa el nivel educativo los retornos sobre el salario aumentan; 4 . El ser mujer reduce en cerca de $5 \%$ la posibilidad de participar en el mercado de trabajo (Anexo 1); 5. Las variables que más explican la participación laboral de los hombres son: la edad, el tener dependientes menores a 6 años, el tener más miembros del hogar desocupados y ser jefe de hogar; mientras que para las mujeres las principales variables explicativas de la participación laboral son la edad y el ser jefe de hogar; 6 . El tener dependientes menores de 6 años y el estar comprometida son las variables que reducen la participación de la mujer en el mercado laboral, aspecto que se encuentra relacionado con la estructura de restricciones.

Por otra parte, con la información de los salarios reales se procede a estimar la diferencia de salarios existente entre hombres y mujeres a partir del modelo de descomposición Oaxaca-Blinder (Blinder, 1973). Con base en esta metodología se pretende observar la diferencia de salarios dada, por una parte, por las variables explicativas observables, especialmente de capital humano $y$, por otra parte, definidas por la parte no observada asociada a la discriminación. Luego, el modelo de capital humano está determinado por la edad, años de educación, experiencia potencial y experiencia potencial al cuadrado; mientras que el modelo de títulos se complementará con las variables dummy de niveles de educación (primaria, secundaria y universidad). Es importante aclarar que el sesgo de selección es corregido bajo la metodología usada por Mora y Caicedo (2013).

De esta forma, la Tabla 10 indica que desde el año 2013 hasta el primer semestre de 2017, la brecha salarial en el modelo de capital humano cayó de 22,72 \% a 13,19 $\%$, mientras que la descomposición no explicada bajó de $28,29 \%$ a $18,39 \%$. Por su parte, el modelo de títulos permite observar que durante el periodo analizado la brecha salarial explicada cayó cinco puntos porcentuales, mientras que la discriminación bajó en cerca de 6 \%. Las anteriores cifras demuestran que, a pesar de existir una reducción en las brechas salariales por sexo, frente a la discriminación -es decir, el componente no explicado- aún persiste este problema que representa 
la existencia de estructuras de restricción en el mercado de trabajo, aspecto que se refleja en términos de remuneraciones a las mujeres.

Tabla 10. Nariño. Descomposición Blinder-Oaxaca en logaritmo del ingreso laboral por hora. 2013-2017-।

\begin{tabular}{|l|c|c|c|c|c|}
\hline \multicolumn{5}{|c|}{ MODELO DE CAPITAL HUMANO } \\
\hline & $\mathbf{2 0 1 3}$ & $\mathbf{2 0 1 4}$ & $\mathbf{2 0 1 5}$ & $\mathbf{2 0 1 6}$ & $\mathbf{2 0 1 7 - I}$ \\
\hline Diferencia salarial (Brecha salarial) & $22,72 \%$ & $10,26 \%$ & $17,23 \%$ & $17,15 \%$ & $13,19 \%$ \\
\hline Descomposición no explicada (Discriminación) & $28,29 \%$ & $15,28 \%$ & $23,81 \%$ & $25,03 \%$ & $18,39 \%$ \\
\hline Modelo de títulos & $\mathbf{2 0 1 3}$ & $\mathbf{2 0 1 4}$ & $\mathbf{2 0 1 5}$ & $\mathbf{2 0 1 6}$ & $\mathbf{2 0 1 7 - I}$ \\
\hline \multicolumn{5}{|c|}{} \\
\hline Diferencia salarial (Brecha salarial) & $23,68 \%$ & $11,09 \%$ & $18,23 \%$ & $19,19 \%$ & $18,58 \%$ \\
\hline Descomposición no explicada (Discriminación) & $30,78 \%$ & $17,28 \%$ & $25,32 \%$ & $27,83 \%$ & $24,95 \%$ \\
\hline
\end{tabular}

Fuente: Cálculos del Observatorio de Género de Nariño a partir de GEIH - DANE.

\section{Discusión}

Hasta el momento se puede afirmar que en Colombia existe una extensa normativa que ha buscado fortalecer el principio de equidad entre hombres y mujeres, regulando las relaciones laborales y las diferencias salariales; sin embargo, como ya se observó en la evidencia empírica, dentro del mercado de trabajo aún persisten grandes problemas de discriminación, los que se encuentran relacionados, entre otros factores, con una estructura de restricción, intrínsecas e impuestas, que limitan la entrada de la mujer al mercado de trabajo. Luego, la persistencia de las brechas de género ha afectado la autonomía económica de la mujer en Nariño, aspecto que representa un reto para el Estado colombiano, especialmente en cuanto a regular de manera más eficiente las relaciones sociales y de género dentro del mercado de trabajo.

Sobre este último aspecto, en Nariño se percibe un crecimiento en la Tasa Global de Participación (TGP) y la tasa de ocupación de las mujeres, escenario que para Kabeer (2012)y Sanchís y Katzkowicz (2014), podría significar un proceso de mayor autonomía económica; empero, dado que los empleos a los que accede la mujer se encuentran relacionados con la informalidad, la precariedad laboral, la segregación ocupacional 
y la discriminación laboral, se puede afirmar que, de acuerdo con Calcagni y Cortínez (2017), existe una gran proporción de mujeres ocupadas que se encuentran lejos de alcanzar su autonomía económica. De ahí, que normas como: la Ley 278 de 1996, la Ley 823 de 2003, la Ley 1429 de 2010, el Decreto 4463 de 2011, la Ley 1496 de 2011 y el Decreto 2733 de 2012, que favorecen el empleo de la mujer, no resuelvan adecuadamente los problemas que este trabajo ha demostrado, pues tener unos ingresos por concepto de trabajo, en condiciones laborales inadecuadas, no es sinónimo de autonomía económica, de empoderamiento, de acceso a recursos y de control sobre los mismos.

En este mismo sentido, la evidencia empírica demostró que existe una mayor salida de mujeres, especialmente jóvenes, al mercado de trabajo. De acuerdo con Panesso, Santacruz, Jaramillo, Ruano y Salazar (2013, p.29), esta situación está relacionada con que "el ciclo productivo de las mujeres es menor que el de los hombres conforme avanzan en edad". Es decir, debido a las restricciones intrínsecas e impuestas (Kabeer, 2012), la mujer tiene más problemas de encontrar trabajo a medida que aumenta su edad, aspecto que está asociado a su rol establecido históricamente dentro de la sociedad, que la relaciona como responsable del cuidado del hogar: cuidado de los hijos, de los ancianos, preparación de los alimentos, aseo de la casa, entre otras actividades que le dificultan insertarse en el mercado de trabajo (Ruiz \& Nicolás, 2018). Luego, a medida que la mujer aumenta de edad, consigue pareja y tiene hijos, su salida al mercado de trabajo -en la mayoría de casos-, es más difícil, pues, por una parte, presenta barreras intrínsecas al interior del hogar, y por otra, cuando logra conseguir un trabajo, presentará una doble o triple carga laboral (Ruiz \& Nicolás, 2018).

Sobre este tema, el Estado colombiano posee dos retos, por una parte, eliminar en el mercado de trabajo las barreras de entrada para las mujeres cabeza de familia, pero también, debe fortalecer el sistema estadístico, con el fin de hacer más visible la "economía oculta" (Díaz, 2002). Sobre el primer tema, si bien existen la Ley 82 de 1993; la Ley 823 de 2003, la Ley 1257 de 2008 y la Ley 1857 de 2017, aún no se han podido erradicar los problemas de discriminación laboral de las mujeres cabeza de hogar, de igual forma, la mujer aún presenta problemas para reducir su carga en el hogar, lo que demuestra una baja armonización entre su familia y su trabajo. En cuanto al segundo tema, es importante reconocer que la Ley 1413 de 2010 permitió el avance, 
aunque incipiente, del desarrollo de una cuenta satélite de la economía del cuidado para Colombia; sin embargo, es necesario consolidar este proceso, avanzar hacia una desagregación regional y departamental, que permita tener una radiografía más efectiva sobre la distribución de tiempos y trabajos en los diferentes territorios.

De igual forma, la evidencia empírica posibilitó analizar la brecha salarial entre hombres y mujeres en Nariño, destacando, a partir del modelo de descomposición, que existe un alto porcentaje que puede ser tomado como discriminación. En este sentido, gran parte de estas brechas responden a la estructura económica y el tejido empresarial, aunque también, se encuentran relacionadas con los factores de segregación vertical y horizontal existentes al interior del mercado laboral. Luego, la feminización del sector terciario y de las ocupaciones ligadas al comercio, ventas y servicios, está relacionada con la precarización laboral, debido a las características que poseen estas actividades u ocupaciones y que, basado en Weller (2004), ya se definió anteriormente. Por ello, si bien la Ley 1496 de 2011 propende por la igualdad salarial y el ingreso de las mujeres a espacios productivos no tradicionales para ellas, se hace necesario que el Estado impulse en mayor medida esta norma, recurriendo a los departamentos y municipios para que desde los territorios se haga más efectiva.

\section{Conclusiones}

A partir del año 1991, Colombia presenta un gran avance en el tema normativo sobre la equidad de género, la mayoría de normas ratifican tratados o convenios internacionales, los que buscan reducir las desigualdades de género, producto de los estereotipos patriarcales, culturalmente heredados. No obstante, a pesar de este avance, dentro del mercado de trabajo de Nariño aún persisten las asimetrías de género. Así, son las mujeres quienes tienen menores tasas de ocupación, mayores porcentajes de desempleo, alta informalidad y menores ingresos. Luego, estas diferencias están relacionadas no solo con las estructuras de restricciones sino también con la estructura económica y el tejido empresarial del departamento.

Según Mayobre (2010), la persistencia de la inequidad de género, a pesar del avance normativo, demuestra que el sistema patriarcal ha disimulado sus efectos discriminatorios. En este sentido, el avance en el tema normativo solo ha producido: 
(...) un cambio superficial, el sistema patriarcal edulcora sus manifestaciones más discriminatorias, se tornan más sutiles los procedimientos discriminatorios, pero no se produce una transformación de su urdimbre más profunda, no se modifica su lógica excluyente, manifestándose la discriminación con un rostro más benevolente, aunque en el fondo los procedimientos de subordinación sigan siendo esencialmente los mismos. (p.2)

Por ello, no es extraño que persistan los problemas relacionados con la participación laboral, la segregación laboral y la discriminación laboral, factores que representan un reto para el Estado colombiano y que deben ser superados si se quiere conseguir un escenario social en el que sea evidente la autonomía económica y empoderamiento de la mujer.

Sobre este aspecto, como ya se anotó en la discusión, es necesaria una mayor presencia del Estado dentro del mercado de trabajo, especialmente para brindar condiciones de contratación y trabajo adecuadas para las mujeres con responsabilidades familiares. Sin embargo, también es importante iniciar a reconocer las actividades invisibilizadas o infravaloradas, de modo que se genere un cambio hacia una sociedad más inclusiva.

Conviene resaltar la importancia del Estado en la búsqueda de la equidad de género; empero, también es necesario reconocer que existen otros actores sociales (sindicatos, instituciones educativas, empresas, organizaciones de mujeres y ONG) que deben ser llamados a aportar en este proceso que busca alcanzar los objetivos previstos en la política pública de la mujer en Colombia. Luego, la lucha por la búsqueda de la autonomía económica de la mujer es un proceso de largo plazo, que debe incluir a la mayor parte de los actores con el fin de romper con los estereotipos de género propios de una sociedad patriarcal.

Los recientes movimientos de mujeres a nivel internacional por el reconocimiento de las actividades de cuidado, refuerzan estas apreciaciones, señalando que solo su valoración económica y las consecuentes obligaciones estatales y sociales frente a las que trabajan en sus hogares, dignificará este trabajo, contribuirá a la equidad en las relaciones de pareja y dará un impulso a su autonomía económica. 
Finalmente debe insistirse en la urgencia de incluir a los hombres en las actividades de cuidado, de aseo, cocina y demás labores domésticas, ya que esta redistribución de los tiempos, incrementará las posibilidades de las mujeres de desarrollar trabajos de tiempo completo y/o de mayor responsabilidad.

\section{Referencias bibliográficas}

Anker, R. (2000). Segregación profesional entre hombres y mujeres: repaso de las teorías. Revista Internacional del Trabajo, 116(3), 343-370.

Bennet, L. (2002). Using Empowerment and Social Inclusion for Pro-poor growth: a Theory of Social Change. Washington, D.C: World Bank.

Blinder, A. (1973). Wage discrimination: Reduced form and structural estimates. The Journal of Human Resources, 8(4), 436-455.

Calcagni, M. y Cortínez, V. (2017). Territorios, empoderamiento y autonomía económica: diversas trayectorias para avanzar en equidad de género en Chile. Serie documento de trabajo $N^{\circ} 223$. Santiago: Rimisp.

Campillo, F. (1999). El trabajo doméstico no remunerado en la economía. Macroeconomía, género y Estado. Bogotá: Departamento Nacional de Planeación, Tercer Mundo Editores.

Carrasco, C. (2005). La economía feminista: una apuesta por otra economía. En: Estudios sobre género y economía. Madrid: Akal Ediciones.

CEPAL, (2014). Desempleo femenino y la autonomía económica de las mujeres. Santiago: Observatorio de igualdad de género de América Latina y el Caribe.

CEPAL, Comisión Económica para América Latina y el Caribe. (2016a). Agenda 2030 y los Objetivos de Desarrollo Sostenible. Una oportunidad para América Latina y el Caribe. Santiago: Publicación de las Naciones Unidas.

CEPAL, (2016b). Autonomía de las mujeres e igualdad en la agenda de desarrollo sostenible. CEPAL: Montevideo.

DANE, Departamento Administrativo Nacional de Estadística (2016a). Ficha Metodológica. Gran Encuesta Integrada de Hogares. Bogotá D.C: DANE.

DANE (2016b). Mercado laboral por departamentos. Bogotá D.C: DANE.

Deere, C. y León, M. (2002). Género, propiedad y empoderamiento: tierra, Estado y mercado en América Latina. México D.F.: Universidad Nacional Autónoma de México-FLACSO (Sede Ecuador). 
Díaz, D. (2002). Situación de la mujer rural colombiana. Perspectiva de género. Cuadernos tierra y justicia. Bogotá: ILSA.

DPS, Departamento para la Prosperidad Social, y Programa de las Naciones Unidas para el Desarrollo (PNUD, 2014). Brechas de género en el mercado laboral. Una metodología para generar información, sensibilizar e incidir. Bogotá D.C: Programa de las Naciones Unidas para el Desarrollo.

Durán, M. A. (2005). Prólogo. En: Estudios sobre género y economía. Madrid: Akal Ediciones.

Espino, A. (2012). Perspectivas teóricas sobre género, trabajo y situación del mercado laboral latinoamericano. En: V. Esquivel(Ed.) La economía feminista desde América Latina: una hoja de ruta sobre los debates actuales en la región (pp.190-246) Santo Domingo: ONU Mujer.

Galvis-Aponte, L. y Pérez-Valbuena, G. (2015). Informalidad laboral y calidad del empleo en la Región Pacífica colombiana. Bogotá: Banco de la República - Economía Regional.

Goyes, I. (2011). Mujer, maternidad y trabajo. Pasto: Universidad de Nariño.

Gobernación de Nariño (2012). Plan de desarrollo 2012-2015 "Nariño Mejor". Pasto: Gobernación de Nariño.

Golla, A., Malhotra, A., Nanda, P. y Mehra, R. (2011). Understanding and measuring women's economic empowerment. Definition, framework, indicators. Washington D.C.: International Centre for Research on Women (ICRW).

Grown, C., Gupta, G. y Zahia K. (2003). Promises to Keep: Achieving Gender Equality and the Empowerment of Women. Washington, D. C.: International Center for Research on Women.

IGAC, Instituto Geográfico Agustín Codazzi (2014). Nariño: características geográficas. Bogotá: IGAC.

Kabeer, N. (2008). Mainstreaming gender in social protection for the informal economy. London: Commonwealth Secretariat.

Kabeer, N. (2012). Women's Economic Empowerment and Inclusive Growth: Labour Markets and Enterprise Development. Ottawa: IDRC, DFID.

Kabeer, N. (2009). Women's Economic Empowerment: Key Issues and Policy Options. Stockholm: Sida Policy. 
León, M. (2001). El empoderamiento de las mujeres: encuentro del primer y tercer mundos en los estudios de género. Revista de Estudios de Género: La Ventana, 2(13), 94-103.

López, D. E. (2005). El derecho de los jueces. Bogotá: LEGIS.

Marco, F. (2015). Entre el ideal de estabilidad laboral y el sueño del negocio propio en Bolivia. La Plata: ONU Mujeres.

Mayobre, P. (2010). Micromachismos invisibles, los otros rostros del patriarcado". Ponencia. VI Congreso Estatal de Isonomía sobre Igualdad entre Mujeres y Hombres, 1-10.

Mora, J. J. y Caicedo, C. (2013). ¿lgualdad salarial entre hombres y mujeres en Santiago de Cali? De la legislación a la realidad. Bogotá D.C: PNUD-DPS-Mintrabajo.

Murguialday, C. (2013). Empoderamiento de las mujeres: conceptualización y estrategias. Barcelona: Icaria.

Ñopo, H. (2012). New Century, old disparities gender and ethnic earnings gaps in Latin America and the Caribbean. Washington DC: Inter-american Development BankWorld Bank.

Panesso, F., Santacruz, A., Jaramillo, V., Ruano, E. y Salazar, Á. (2013). Brechas de género en el mercado de trabajo de Pasto. Bogotá D.C.: Programa de las Naciones Unidas para el Desarrollo (PNUD).

Pérez, A. (2005). "Economía del género y economía feminista, ¿conciliación o ruptura? Revista Venezolana de Estudios de la Mujer, 10(24), 43-64.

PNUD (2012). Regiones en conflicto. Comprender para transformar. Bogotá: Naciones Unidas.

PNUD, Programa de las Naciones Unidas para el Desarrollo (2009). El departamento de Nariño frente a los Objetivos de Desarrollo del Milenio. San Juan de Pasto: Naciones Unidas.

Ramírez, J., Zambrano, M., Mogrovejo, J. y Carreño, J. (2016). Informalidad laboral en los departamentos de Norte de Santander, Nariño, La Guajira y Cesar. Apuntes del CENES, 35(62), 125-145.

Ramos, D. (2014). Violencia de género en Colombia. Mito. Revista Cultural.

Red Ormet (2015). ANEXO 1 - ¿Qué es el factor de expansión?

Ruiz, I. \& Nicolás, M. (2018). La cuidadora familiar: sentimiento de obligación naturalizado de la mujer a la hora de cuidar. Enfermería Global, 1749), 420-447. 
Sanchís, N. y Katzkowicz, N. (2014). Argentina. Obstáculos para la inserción laboral equitativa y el empoderamiento económico de las mujeres. Buenos Aires: Pnud-Aecid.

Sarmiento, L. y Vargas, H. (2002). El trabajo de las mujeres en Colombia. Bogotá: Corporación Viva la Ciudadanía.

Vara, M. J. (2005). "Empleo femenino en las cadenas de la producción global". En: Estudios sobre género y economía. Madrid: Akal Ediciones.

Vega, C. y Vásconez, A. (2016). Mujeres, empoderamiento y trabajo informal en Ecuador. Diversidad de regímenes y restricción de derechos. Quito: ONU Mujeres.

Weller, J. (2004). El empleo terciario en América Latina: entre la modernidad y la sobrevivencia". Revista de la CEPAL 84, 159-176. 


\section{Anexos}

Anexo 1. Nariño. Modelo de probabilidad de participación laboral. Efectos marginales

\begin{tabular}{|c|c|c|c|c|c|}
\hline VARIABLES & 2013 & 2014 & 2015 & 2016 & 2017-I \\
\hline \multirow{2}{*}{ Edad } & $0,0269 * * *$ & $0,0264^{* * *}$ & $0,0236^{* * *}$ & $0,0247^{* * *}$ & $0,0217^{* * *}$ \\
\hline & $(0,000911)$ & $(0,000934)$ & $(0,000821)$ & $(0,000905)$ & $(0,00127)$ \\
\hline \multirow{2}{*}{ Edad al cuadrado } & $-0,0003^{* * *}$ & $-0,0003^{* * *}$ & $-0,00029 * * *$ & $-0,00030^{* * *}$ & $-0,0002^{* * *}$ \\
\hline & $(1,02 e-05)$ & $(1,02 e-05)$ & $(9,14 \mathrm{e}-06)$ & $(1,04 e-05)$ & $(1,40 e-05)$ \\
\hline \multirow{2}{*}{ Años de educación } & $0,00891^{* * *}$ & $0,0094^{* * *}$ & $0,00978^{* * *}$ & $0,00818^{* * *}$ & $0,00854^{* * *}$ \\
\hline & $(0,000821)$ & $(0,000859)$ & $(0,000803)$ & $(0,000699)$ & $(0,00112)$ \\
\hline \multirow{2}{*}{$\begin{array}{l}\text { Familiares menores } \\
\text { de } 6 \text { años }\end{array}$} & $0,0157^{* * *}$ & 0,00560 & $0,0139 * * *$ & $0,0110^{* * *}$ & 0,00514 \\
\hline & $(0,00393)$ & $(0,00373)$ & $(0,00386)$ & $(0,00366)$ & $(0,00541)$ \\
\hline \multirow{2}{*}{$\begin{array}{l}\text { Familiares mayores } \\
\text { de } 6 \text { años }\end{array}$} & 0,00480 & 0,00476 & 0,00497 & $0,0123^{* * *}$ & $0,0150^{* * *}$ \\
\hline & $(0,00359)$ & $(0,00347)$ & $(0,00333)$ & $(0,00320)$ & $(0,00424)$ \\
\hline \multirow{2}{*}{ Riqueza } & $-0,0320^{* * *}$ & $-0.0267^{* * *}$ & $-0,0241^{* * *}$ & $-0,0200^{* * *}$ & $-0,0193^{* * *}$ \\
\hline & $(0,00327)$ & $(0,00320)$ & $(0,00284)$ & $(0,00267)$ & $(0,00360)$ \\
\hline \multirow{2}{*}{ Otros ingresos } & $-5,42 e-09 * *$ & $-3,79 e-09$ & $\begin{array}{l}-5,53 e- \\
09^{* * *}\end{array}$ & $\begin{array}{l}-6,00 e- \\
09^{* * *}\end{array}$ & $2,14 e-09$ \\
\hline & $(2,15 e-09)$ & $(2.54 \mathrm{e}-09)$ & $(1,72 e-09)$ & $(1,78 e-09)$ & $(1,87 e-09)$ \\
\hline \multirow{2}{*}{$\begin{array}{l}\text { Otros desempleados } \\
\text { en el hogar }\end{array}$} & $0,0237^{* * *}$ & $0,0420 * * *$ & $0,0529 * * *$ & $0,0158^{* * *}$ & $0,0308^{* * *}$ \\
\hline & $(0,00592)$ & $(0,00673)$ & $(0,00743)$ & $(0,00556)$ & $(0,00957)$ \\
\hline \multirow{2}{*}{ Sexo (1=Mujer) } & $-0,0670^{* * *}$ & $-0,0617^{* * *}$ & $-0,0406^{* * *}$ & $-0,0587^{* * *}$ & $-0,0324^{* * *}$ \\
\hline & $(0,00669)$ & $(0,00669)$ & $(0,00579)$ & $(0,00599)$ & $(0,00769)$ \\
\hline \multirow{2}{*}{ Compromiso (1=Si) } & $-0,00809$ & $-0.0117^{* *}$ & $-0,00490$ & $-0,0139 * * *$ & $-0,00945$ \\
\hline & $(0,00557)$ & $(0,00566)$ & $(0,00552)$ & $(0,00496)$ & $(0,00719)$ \\
\hline \multirow{2}{*}{ Jefe de hogar (1=Si) } & $0,0150^{* *}$ & $0,0184^{* *}$ & $0,0255^{* * *}$ & $0,0218^{* * *}$ & $0,0271^{* *}$ \\
\hline & $(0,00736)$ & $(0,00773)$ & $(0,00753)$ & $(0,00718)$ & $(0,0105)$ \\
\hline Observations & 10,671 & 10,710 & 10,692 & 10,331 & 5,014 \\
\hline
\end{tabular}

Robust standard errors in parentheses

${ }^{* * *} p<0.01,{ }^{* *} p<0.05,{ }^{*} p<0.1$

Fuente: Cálculos del Observatorio de Género de Nariño a partir de GEIH - DANE. 
Anexo 2. Metodología de investigación cuantitativa

La metodología de tipo cuantitativo partirá de la identificación de las brechas sexuales en los principales indicadores laborales, seguida de un análisis sobre las brechas salariales. Para ello, se realizan varios análisis de diferencias de medias, y después enfocarse en las brechas salariales y el proceso de inserción laboral. Para estos últimos se utilizará inicialmente el modelo de Heckman con corrección de sesgo de selección (Mora \& Caicedo, 2013) para análisis de los retornos sobre el ingreso y se proseguirá con análisis de la descomposición de diferencias para medición de discriminación desde el modelo de Oaxaca-Blinder (Blinder, 1973).

Los datos para este trabajo fueron tomados de la Gran Encuesta Integrala de Hogares $(G E I H)$, donde se procedió a empalmar los datos anuales con el fin de obtener un proxy departamental (Red Ormet, 2015; DANE, 2016). Luego, el uso de la GEIH es adecuado, puesto que es una encuesta que posibilita profundizar sobre el objetivo que este articulo persigue, dado que su interés es preguntar sobre las condiciones de empleo de las personas, las características socioeconómicas de la población y las características de sus ingresos. 